\title{
Vanishing and nilpotence of locally trivial symmetric spaces over regular schemes
}

\author{
Paul Balmer
}

\begin{abstract}
We prove two results about Witt rings $\mathrm{W}(-)$ of regular schemes. First, given a semi-local regular ring $R$ of Krull dimension $d$, if $U$ is the punctured spectrum, obtained from $\operatorname{Spec}(R)$ by removing the maximal ideals of height $d$, then the natural map $\mathrm{W}(R) \rightarrow \mathrm{W}(U)$ is injective. Secondly, given a regular integral scheme $X$ of finite Krull dimension, consider $Q$ its function field and the natural map $\mathrm{W}(X) \rightarrow \mathrm{W}(Q)$. We prove that there is an integer $N$, depending only on the Krull dimension of $X$, such that the product of any choice of $N$ elements in $\operatorname{Ker}(\mathrm{W}(X) \rightarrow \mathrm{W}(Q))$ is zero. That is, this kernel is nilpotent. We give upper and lower bounds for the exponent $N$.
\end{abstract}

Mathematics Subject Classification (2000). 19G12 (11E81, 18E30).

Keywords. Witt group of schemes, rational kernel, semi-local ring, triangular Witt groups.

\section{Introduction}

In [9], Knebusch defined the Witt group $\mathrm{W}(X)$ of an arbitrary scheme $X$. It is the quotient of the abelian monoid of symmetric spaces up to isometry by the submonoid of metabolic spaces. The tensor product turns $\mathrm{W}(X)$ into a commutative ring with unit.

Assume in what follows that $X$ is integral with function field $Q$ and that 2 is everywhere invertible on $X$. Let us consider the natural map $\mathrm{W}(X) \rightarrow \mathrm{W}(Q)$. This map is known not to be injective in general, even when $X$ is affine and regular, see for instance Knus [10, Example 2.5.3, p. 480]. A very old conjecture says: $\mathrm{W}(R) \rightarrow \mathrm{W}(Q)$ is injective when $R$ is regular local, see for instance [9, p. 256]. So, for convenience, we shall refer to it as Knebusch's conjecture.

This conjecture is known to hold when $R$ contains a field, see OjangurenPanin [11, Theorem A]. The general case is still open, but known to be true when $\operatorname{dim}(R) \leq 4$, see [4, Corollary 10.4]. Here, we shall extend this low dimension result to $R$ semi-local, see Corollary 3.6. This will follow from the first main result of this paper, true in any dimension: 
Theorem. Let $R$ be a noetherian semi-local regular ring containing $\frac{1}{2}$. Let $U$ be the punctured spectrum, obtained from $\operatorname{Spec}(R)$ by removing the maximal ideals of maximal height. Then the natural homomorphism $\mathrm{W}(R) \rightarrow \mathrm{W}(U)$ is injective. (See Theorem 3.3 below.)

Our second theme is the kernel $\operatorname{Ker}(\mathrm{W}(X) \rightarrow \mathrm{W}(Q))$, for a global scheme $X$, noetherian and regular. It is known since Knebusch [9], that its elements are nilpotent in $\mathrm{W}(X)$. Actually, in the affine case, the result is due to Craven-RosenbergWare [6, Theorem 2.8], and the global case follows easily from [9, Corollary p. 185]. If $X$ is of Krull dimension at most 3, Charles Walter and the author proved that this kernel is zero, see [4, Corollary 10.3]. The first non-zero kernel appears in dimension 4. Here we improve the Craven-Rosenberg-Ware-Knebusch nilpotence result as follows:

Theorem. Let $X$ be a noetherian separated regular integral scheme containing $\frac{1}{2}$. Let $Q$ be its function field. Assume that $X$ is of finite Krull dimension. Then there exists an integer $N$ depending only on $\left[\frac{\operatorname{dim}(X)}{4}\right]$ such that the $N$-th power of the ideal $\operatorname{Ker}(\mathrm{W}(X) \rightarrow \mathrm{W}(Q))$ is zero. For $0 \leq \operatorname{dim}(X) \leq 3$, we have $N=1$ and for $4 \leq \operatorname{dim}(X) \leq 7$, we have $N=2$. (See Corollary 4.3 and Theorem 4.5.)

In general, we can take $N=2^{\left[\frac{\operatorname{dim} X}{4}\right]}$. If moreover the conjectural injectivity $\mathrm{W}\left(\mathcal{O}_{X, x}\right) \hookrightarrow \mathrm{W}(Q)$ holds for any local ring of $X$, then we can take the exponent $N$ to be $\left[\frac{\operatorname{dim} X}{4}\right]+1$. Observe that both coincide up to dimension 7 included. We prove also, by giving examples, that we cannot expect a better general bound than $N=\left[\frac{\operatorname{dim} X}{4}\right]+1$, see Corollary 5.3.

Both results are classical, in the sense that they can already be stated in the 1976 language introduced by Knebusch. Their proofs are triangular, in the sense that they rely on the series triangular Witt groups [1], [2], where Witt groups of triangulated categories are introduced and some of their basic properties are established. These techniques have already brought interesting classical applications as can be seen in [3] and [4]. For the convenience of the reader, we included in Section 2 a short introduction to this material. We shall also make use of the multiplicative structure on these triangular Witt groups, induced by the tensor product. Details on multiplicative structures can be found in Gille and Nenashev [8].

In this triangular Witt theory, we have not only one but several Witt groups $\mathrm{W}^{n}(X)$ for $n \in \mathbb{Z}$, in a 4-periodic way: $\mathrm{W}^{n+4} \simeq \mathrm{W}^{n}$. The multiplicative structure on the total Witt group $\mathrm{W}(X) \oplus \mathrm{W}^{1}(X) \oplus \mathrm{W}^{2}(X) \oplus \mathrm{W}^{3}(X)$ makes it a $\mathbb{Z} / 4$ graded ring. We also prove in the last Section that the "new" part $\mathrm{W}^{1}(X) \oplus$ $\mathrm{W}^{2}(X) \oplus \mathrm{W}^{3}(X)$ is nilpotent in this total Witt ring, still for $X$ regular of finite Krull dimension, see Proposition 6.1.

Acknowledgments. I hope that Stefan Gille, Michel Matthey, Manuel Ojan- 
guren, Winfried Scharlau and Charles Walter will accept my sincere thanks for their precious and friendly support.

\section{Triangular Witt groups for the working mathematician}

The basic concepts of "triangulated categories with duality", of "Witt groups" of such objects, of "shifted dualities", and so on, are to be found in the series triangular Witt groups [1] and [2]. These two articles include many details and should provide an easy introduction to this material. We only give here a quick overview in order to make the reading of the present article easier.

A triangulated category $K$ is an additive category, equipped with a translation $T: K \stackrel{\sim}{\rightarrow} K$, in which we have chosen a collection of exact triangles, which are taking the role played by exact sequences in exact or abelian categories. The foundations are due to Grothendieck and Verdier [13], a good introduction can be found in Weibel [15, Chapter 10], and a minimal baggage can be acquired in [1, Section 1], where the enriched octahedron axiom is stated. This axiom is due to Beilinson, Bernstein and Deligne [5, 1.1.14, p. 26], is true in all known triangulated categories, and is always assumed below without further notice. The basic example of a triangulated category is the (bounded) derived category of an exact category $\mathcal{E}$, that is the category of bounded complexes over $\mathcal{E}$, with morphisms obtained from homotopy classes of morphisms of complexes by inverting quasi-isomorphisms.

A duality \# on a triangulated category $K$ is a contravariant functor $\#: K^{o p} \rightarrow$ $K$ which is exact (respects triangles) and is an involution $\operatorname{Id} \simeq(\#)^{2}$. We can define the Witt group of a triangulated category with duality almost by the usual procedure, namely we consider the monoid of symmetric spaces (pairs $(E, \varphi)$ where $\varphi=\varphi^{\#}: E \stackrel{\sim}{\rightarrow} E^{\#}$ is a symmetric isomorphism) and divide out the metabolic spaces, rather called neutral spaces when we have to distinguish the triangular and classical frameworks. These neutral spaces are those possessing a Lagrangian, no surprise about that, except that this definition must involve exact triangles where we classically used exact sequences. See a detailed and precise definition in $[1$, Section 2]. The reader who wants to refresh his knowledge of classical Witt groups can browse [9, Chapter I] for schemes or [2, Section 1] for the obvious extension to exact categories. We connect the classical and triangular Witt groups as follows.

Definition 2.1. Let $X$ be a scheme. We denote here by

$$
\mathrm{D}^{\mathrm{b}}(X)
$$

the derived category of bounded complexes of vector bundles over $X$. (This sloppy notation, not dwelling on the choice of vector bundles as opposed to other classes of $\mathcal{O}_{X}$-modules, is of no harm over noetherian separated regular schemes as we shall use below. Better but heavier is $\mathrm{D}^{\mathrm{b}}\left(\mathrm{VB}_{X}\right)$.) The triangulated category $\mathrm{D}^{\mathrm{b}}(X)$ is equipped with a duality, derived from $\mathcal{H}_{0} m_{\mathcal{O}_{X}}\left(-, \mathcal{O}_{X}\right)$. We have a 
natural homomorphism $\mathrm{W}(X) \rightarrow \mathrm{W}\left(\mathrm{D}^{\mathrm{b}}(X)\right)$ which sends a symmetric space to the complex concentrated in degree zero with the obvious symmetric form. This is an isomorphism.

Theorem 2.2. Let $X$ be a scheme on which 2 is everywhere invertible, then the natural homomorphism $\mathrm{W}(X) \rightarrow \mathrm{W}\left(\mathrm{D}^{\mathrm{b}}(X)\right)$ described above is an isomorphism.

This agreement between the usual and the "derived" Witt groups holds more generally for exact categories with duality and is the main result of [2]. Triangular Witt groups were introduced in order to study the behaviour of Witt groups with respect to localization. In its most abstract and general form, the Localization Theorem is the following.

Theorem 2.3. Let $J \longmapsto K \rightarrow L$ be an exact sequence of triangulated categories with duality in which 2 is invertible. Then there is a long exact sequence of Witt groups:

$$
\cdots \rightarrow \mathrm{W}^{-1}(L) \rightarrow \mathrm{W}^{0}(J) \rightarrow \mathrm{W}^{0}(K) \rightarrow \mathrm{W}^{0}(L) \rightarrow \mathrm{W}^{1}(J) \rightarrow \cdots
$$

which is 12-term periodic.

This Theorem is the main result of [1]. In the above, a short exact sequence of triangulated categories with duality $J \longmapsto K \rightarrow L$ means that $J$ is a full triangulated subcategory of $K$ which is saturated $(E \oplus F \in J \Rightarrow E, F \in J)$ and $L$ is the quotient of $K$ by $J$; moreover $J, K$ and $L$ carry dualities in a compatible way.

Remark 2.4. The shifted Witt groups $\mathrm{W}^{n}, n \in \mathbb{Z}$, which appear in this long exact sequence are simply obtained by shifting the duality, that is essentially replacing the duality \# by $T^{n} \circ \#$. They are 4-periodic: $\mathrm{W}^{n} \cong \mathrm{W}^{n+4}$ and $\mathrm{W}^{0}$ is the above $\mathrm{W}$. We warn the careful reader that this overview hides the existence and importance of signs, which appear in the "shifted dualities". Precise definitions are to be found in [1, Section 2].

Hypothesis 2.5. In what follows, we shall consider regular schemes, by which we mean schemes which are separated noetherian and regular. We shall also say that a scheme contains $\frac{1}{2}$, when 2 is invertible in the global sections of the structure sheaf.

Definition 2.6. Let $X$ be a regular scheme and $U$ be an open subscheme. Let $Z$ be the closed complement of $U$ in $X$. We have an exact sequence of triangulated categories with duality: $\mathrm{D}_{Z}^{\mathrm{b}}(X) \mapsto \mathrm{D}^{\mathrm{b}}(X) \rightarrow \mathrm{D}^{\mathrm{b}}(U)$ where $\mathrm{D}_{Z}^{\mathrm{b}}(X)$ is by definition the kernel of the localization $\mathrm{D}^{\mathrm{b}}(X) \rightarrow \mathrm{D}^{\mathrm{b}}(U)$. We denote by $\mathrm{W}_{Z}^{n}(X)$ the $n$-th Witt group of $\mathrm{D}_{Z}^{\mathrm{b}}(X)$, called the $n$-th Witt group of $X$ with supports in $Z$. More on this Witt cohomology theory and its use has appeared in [3]. This subcategory 
$\mathrm{D}_{Z}^{\mathrm{b}}(X)$ is a $\otimes$-ideal in the sense that, for any $E, F \in \mathrm{D}^{\mathrm{b}}(X)$, if $E$ or $F$ belongs to $\mathrm{D}_{Z}^{\mathrm{b}}(X)$ so does $E \otimes F$.

Construction 2.7. Let $X$ be a noetherian scheme. For any complex $E \in \mathrm{D}^{\mathrm{b}}(X)$ let us denote by $\operatorname{Supph}(E)$ the support of the homology of $E$, that is the following closed subset of $X:\left\{x \in X \mid E_{x} \nsucceq 0\right.$ in $\left.\mathrm{D}^{\mathrm{b}}\left(\mathcal{O}_{X, x}\right)\right\}$. For $k \geq 0$, consider the full subcategory of $\mathrm{D}^{\mathrm{b}}(X)$ on the following objects:

$$
\mathrm{D}^{(k)}(X):=\left\{E \in \mathrm{D}^{\mathrm{b}}(X) \mid \operatorname{codim}(\operatorname{Supph}(E)) \geq k\right\} .
$$

We have a decreasing filtration $\mathrm{D}^{\mathrm{b}}(X)=\mathrm{D}^{(0)}(X) \supset \mathrm{D}^{(1)}(X) \supset \mathrm{D}^{(2)}(X) \supset \cdots$ of $\mathrm{D}^{\mathrm{b}}(X)$. If we assume that $X$ has finite Krull dimension $d$, then the filtration ends with zeros $\cdots \supset \mathrm{D}^{(d)}(X) \supset \mathrm{D}^{(d+1)}(X)=\mathrm{D}^{(d+2)}(X)=\cdots=0$. Since triangular Witt groups form a cohomology theory, this leads to the so-called Gersten Witt spectral sequence as studied in [4]. We unfold here some building blocks of this spectral sequence, that we shall use below.

Notations 2.8. If $X$ is a scheme, we denote by $X^{(k)}$ the points of $X$ such that $\mathcal{O}_{X, x}$ is of Krull dimension $k$. We denote the residue field at $x$ by $\kappa(x)$. Both notations are standard.

Theorem 2.9. (Balmer-Walter). Let $X$ be a regular scheme containing $\frac{1}{2}$. Let $k \geq 0$ be an integer. The category $\mathrm{D}^{(k+1)}(X)$ is a saturated subcategory of $\mathrm{D}^{(k)}(X)$ and the quotient $\mathrm{D}^{(k)}(X) / \mathrm{D}^{(k+1)}(X)$ is naturally equivalent to $\coprod_{x \in X^{(k)}} \mathrm{D}^{\mathrm{b}}\left(\mathcal{O}_{X, x}\right.$ - fin.leng. $)$ where we denote by " $\mathcal{O}_{X, x}$ - fin.leng." the abelian category of finite length $\mathcal{O}_{X, x}$-modules. Moreover this induces the following isomorphisms:

$$
\mathrm{W}^{n}\left(\mathrm{D}^{(k)}(X) / \mathrm{D}^{(k+1)}(X)\right) \simeq\left\{\begin{array}{cl}
\bigoplus_{x \in X^{(k)}} \mathrm{W}(\kappa(x)) & \text { for } n \equiv k \bmod 4 \\
0 & \text { otherwise }
\end{array}\right.
$$

This is [4, Proposition 7.1 and Theorem 6.1]. For a point $x \in X^{(k)}$, the duality on the abelian category $\mathcal{O}_{X, x}$ - fin.leng. is given by $\operatorname{Ext}^{k}\left(-, \mathcal{O}_{X, x}\right)$. We shall not use the above result in full strength and in particular, we should not worry about the explicit isomorphism $\mathrm{W}(\kappa(x)) \stackrel{\sim}{\rightarrow} \mathrm{W}\left(\mathcal{O}_{X, x}\right.$ - fin.leng.), which includes a choice of local parameters. The main thing we use is that the $\mathrm{W}\left(\mathcal{O}_{X, x}\right)$-module $\mathrm{W}\left(\mathcal{O}_{X, x}\right.$-fin.leng. $)$ is generated by any form on the finite length $\mathcal{O}_{X, x}$-module $\kappa(x)$. 
Corollary 2.10. With the above notations, for any integer $k \geq 0$, we have a surjection

$$
\mathrm{W}\left(\mathrm{D}^{(4 k+4)}(X)\right) \rightarrow \mathrm{W}\left(\mathrm{D}^{(4 k+3)}(X)\right) \stackrel{\sim}{\rightarrow} \mathrm{W}\left(\mathrm{D}^{(4 k+2)}(X)\right) \stackrel{\sim}{\rightarrow} \mathrm{W}\left(\mathrm{D}^{(4 k+1)}(X)\right) .
$$

Proof. Out of Theorem 2.3, we get long exact sequences associated to the short exact sequences $\mathrm{D}^{(j+1)}(X) \longmapsto \mathrm{D}^{(j)}(X) \rightarrow \mathrm{D}^{(j)}(X) / \mathrm{D}^{(j+1)}(X)$. Using the vanishing of the Witt groups of $\mathrm{D}^{(j)}(X) / \mathrm{D}^{(j+1)}(X)$ for the suitable values of $j$, we get the above result.

Remark 2.11. The last thing we shall make use of is the multiplicative structure which is induced on all the Witt groups in sight by the (derived) tensor product. This is actually slightly more subtle that one can think, in particular for shifted Witt groups, where there exists a left and a right product which differ up to signs. A careful and general exposition of this can be found in Gille and Nenashev [8]. These signs do not appear in $\mathrm{W}^{0}$, the unshifted Witt group, where the reader can follow his classical intuition. The essential thing we shall use below is that the product of the Witt class of a symmetric space $(E, \varphi)$ with the class of a symmetric space $(F, \psi)$ will always be defined on some complex quasiisomorphic to $E \otimes F$. This is enough for our purposes, because we shall only use $\operatorname{Supph}(E \otimes F) \subset \operatorname{Supph}(E) \cap \operatorname{Supph}(F)$ - we even have equality. It is also true that the multiplicative structure gives a $\mathrm{W}^{0}(K)$-module structure to all groups appearing in the localization long exact sequence of Theorem 2.3, when of course $K$ is equipped with a reasonable tensor product and $J$ is a $\otimes$-ideal. The homomorphisms in the long exact sequence are all $\mathrm{W}^{0}(K)$-linear, including the connecting homomorphisms. See if necessary [8, Section 3.2].

Example 2.12. Let $R$ be a commutative ring and let $x \in R$. Consider the Koszul complex $\operatorname{Kos}(x):=\cdots \rightarrow 0 \rightarrow R \stackrel{x}{\longrightarrow} R \rightarrow 0 \rightarrow \cdots$, with $R$ in degree 1 and 0 . Then $\operatorname{Kos}(x)$ has a natural symmetric form for the shifted duality $T^{1} \circ$ \#, see Remark 2.4 for signs. Tensoring $n$ such complexes produces the usual Koszul complex, with its symmetric form for the $n$-th shifted duality. If $R$ is moreover local regular of dimension $n$ and $x_{1}, \ldots, x_{n}$ is a system of parameters generating the maximal ideal, then the Koszul complex over $x_{1}, \ldots, x_{n}$ maps into $\mathrm{D}^{(n)}(R) \simeq \mathrm{D}^{\mathrm{b}}$ (R-fin.leng.) on the residue field $\kappa$, concentrated in degree 0 , and therefore the form obtained above defines a form on $\kappa$. When $n \equiv 0 \bmod 4$, this form is a generator of the $\mathrm{W}(R)$-module $\mathrm{W}(R$-fin.leng. $)$, since the latter is isomorphic to $\mathrm{W}(\kappa)$, see 2.9 and after.

Back to an arbitrary commutative ring $R$, observe that the class in $\mathrm{W}^{1}(R)$ of the form on $\operatorname{Kos}(x)$ is zero, because the form is an isomorphism in each degree (see [2, Remark 5.8]), or simply and directly because the complex having $R$ concentrated 
in degree 0 is a Lagrangian. Therefore, the same vanishing holds in $\mathrm{W}^{n}$ for the symmetric space on $\operatorname{Kos}\left(x_{1}, \ldots, x_{n}\right)=\operatorname{Kos}\left(x_{1}\right) \otimes \cdots \otimes \operatorname{Kos}\left(x_{n}\right)$, the product of zero classes being zero. The reader might be puzzled down by the attention paid to a symmetric space which is in the end zero in the Witt group. Observe that $\operatorname{Kos}\left(x_{1}, \ldots, x_{n}\right)$ can actually make sense in other triangulated categories than $\mathrm{D}^{\mathrm{b}}(R)$, for instance in $\mathrm{D}_{Z}^{\mathrm{b}}(R)$ for any closed $Z \supset V\left(<x_{1}, \ldots, x_{n}>\right)$. In the latter, the above Lagrangian cannot be used because the complex $R$ (concentrated in degree 0) is of course not in $\mathrm{D}_{Z}^{\mathrm{b}}(R)$ unless $Z=\operatorname{Spec}(R)$. In other words, the Witt class of $\operatorname{Kos}\left(x_{1}, \ldots, x_{n}\right)$ in $\mathrm{W}_{Z}^{n}(R)$ will not necessarily be zero, although it goes to zero in $\mathrm{W}^{n}(R)$, via the natural homomorphism $\mathrm{W}_{Z}^{n}(R) \rightarrow \mathrm{W}^{n}(R)$. We shall use this in the proof of Theorem 3.3.

\section{The Witt group of the punctured spectrum in the semi-local case}

In this Section, we denote by $R$ a noetherian semi-local regular ring in which 2 is invertible.

Remark 3.1. Let $\mathfrak{m}$ be a maximal ideal of $R$. Recall that the natural homomorphism $\mathrm{W}(R) \rightarrow \mathrm{W}(\kappa(\mathfrak{m}))$ is surjective. In fact, since any form over $\kappa(\mathfrak{m})$ is diagonalizable, it is enough to see that the homomorphism of the groups of units $R^{\times} \rightarrow \kappa(\mathfrak{m})^{\times}$is surjective. This is an immediate consequence of the Chinese Remainder Theorem, since $R$ has only finitely many maximal ideals.

Lemma 3.2. Let $R$ be a noetherian semi-local regular ring. Let $\mathfrak{m}$ be a maximal ideal of height $n \geq 1$. Then there exists $x_{1}, \ldots, x_{n} \in \mathfrak{m}$ such that:

(1) in the local ring $R_{\mathfrak{m}}$ the sequence $x_{1}, \ldots, x_{n}$ is a system of parameters;

(2) if $\mathfrak{p}$ is any prime ideal of $R$ such that $x_{1}, \ldots, x_{n} \in \mathfrak{p}$ then $\mathfrak{p}=\mathfrak{m}$.

Proof. Consider the ideal $I:=\mathfrak{m}_{1}^{2} \cdot \mathfrak{m}_{2} \cdot \ldots \cdot \mathfrak{m}_{r}$ where $\mathfrak{m}_{1}=\mathfrak{m}, \mathfrak{m}_{2}, \ldots, \mathfrak{m}_{r}$ are all the maximal ideals of $R$. Consider the module $R / I \simeq R / \mathfrak{m}^{2} \times \prod_{j=2}^{r} R / \mathfrak{m}_{j}$. Choose $v_{1}, \ldots, v_{n}$ a $R / \mathfrak{m}$-basis of $\mathfrak{m} / \mathfrak{m}^{2} \subset R / \mathfrak{m}^{2}$. For each $i=1, \ldots, n$ define $x_{i} \in \mathfrak{m}$ such that $x_{i} \equiv v_{i} \bmod \mathfrak{m}^{2}$ and $x_{i} \equiv 1 \bmod \mathfrak{m}^{\prime} \neq \mathfrak{m}$. These $x_{1}, \ldots, x_{n}$ will do.

Theorem 3.3. Let $R$ be a noetherian semi-local regular ring which contains $\frac{1}{2}$ and let $\mathfrak{m}_{1}, \ldots \mathfrak{m}_{r}$ be the maximal ideals of maximal height. Consider the "punctured spectrum", that is the open subscheme $U=\operatorname{Spec}(R) \backslash\left\{\mathfrak{m}_{1}, \ldots \mathfrak{m}_{r}\right\}$. Then the natural map $\mathrm{W}(R) \rightarrow \mathrm{W}(U)$ is injective.

More precisely, for any $n \in \mathbb{Z}$, the natural map $\mathrm{W}^{n}(R) \rightarrow \mathrm{W}^{n}(U)$ is injective and is an isomorphism except when $\operatorname{dim}(R) \equiv n+1 \bmod 4$. When $\operatorname{dim}(R) \equiv n+1$ 
mod 4, we have an exact sequence:

$$
0 \rightarrow \mathrm{W}^{n}(R) \rightarrow \mathrm{W}^{n}(U) \rightarrow \bigoplus_{i=1}^{r} W\left(R / \mathfrak{m}_{i}\right) \rightarrow 0
$$

Proof. Let $d:=\operatorname{dim}(R)$ be the Krull dimension of $R$. Consider the short exact sequence of triangulated categories $\mathrm{D}^{(d)}(R) \longmapsto \mathrm{D}^{\mathrm{b}}(R) \rightarrow \mathrm{D}^{\mathrm{b}}(U)$. (See notations in 2.7.) We obtain a long exact sequence in which, by Theorem 2.9, the Witt groups of $\mathrm{D}^{(d)}(R)$ can be replaced 3 times out of four by zero and the fourth time by $\oplus_{i=1}^{r} W\left(R / \mathfrak{m}_{i}\right)$. This changes according to $d \bmod 4$. With this, it is easy to reduce the Theorem to the fact that the map

$$
\mathrm{W}^{n}\left(\mathrm{D}^{(d)}(R)\right) \rightarrow \mathrm{W}^{n}(R)
$$

is zero. To see this, we can assume that $n \equiv d \bmod 4$ otherwise the lefthand group is already zero, again by Theorem 2.9 , which also tells us that when $n \equiv d$ $\bmod 4, \mathrm{~W}^{n}\left(\mathrm{D}^{(d)}(R)\right) \simeq \oplus_{i=1}^{r} W\left(R_{\mathfrak{m}_{i}}\right.$ - fin.leng. $)$. It suffices to show that the map $\mathrm{W}\left(R_{\mathfrak{m}_{i}}\right.$ - fin.leng. $) \rightarrow \mathrm{W}^{d}(R)$ is zero for each $i=1, \ldots, r$. For this, recall that this map is $\mathrm{W}(R)$-linear and that $\mathrm{W}\left(R_{\mathfrak{m}_{i}}\right.$ - fin.leng. $)$ is generated as a $\mathrm{W}(R)$-module by any class on the finite length module $R / \mathfrak{m}_{i}$, see 2.9 and after, as well as Remark 3.1. So it suffices to show that the image of one symmetric form on the finite length $R_{\mathfrak{m}_{i}}$-module $R / \mathfrak{m}_{i}$ is zero in $\mathrm{W}^{d}(R)$. Let us assume that $i=1$ to fix the ideas.

In other words, the result will follow once we have found a complex $E \in \mathrm{D}^{\mathrm{b}}(R)$ carrying a symmetric form $\varphi$ for the $d$-th shifted duality, and such that:

(1) The complex $E$ belongs to $\mathrm{D}^{(d)}(R)$;

(2) Via the equivalence $\mathrm{D}^{(d)}(R) \simeq \coprod_{i=1}^{r} \mathrm{D}^{\mathrm{b}}\left(R_{\mathfrak{m}_{i}}\right.$ - fin.leng. $), E$ maps to $R / \mathfrak{m}_{1}$ (and to zero for $i=2, \ldots, r$ );

(3) The Witt class $[E, \varphi]$ is zero in $\mathrm{W}^{d}(R)$.

Now we use the above Lemma to choose $x_{1}, \ldots, x_{d} \in R$ such that they form a system of parameters in $R_{\mathfrak{m}_{1}}$ and such that the only prime ideal containing all of them is $\mathfrak{m}_{1}$. Consider the Koszul complex $E=\operatorname{Kos}\left(x_{1}, \ldots, x_{d}\right)$ over $x_{1}, \ldots, x_{d}$ in $R$ with its usual symmetric form for the $d$-th shifted duality, as described in Example 2.12. It is clear from the Lemma, that $E$ satisfies properties (1) and (2) above. To see (3), just give an explicit Lagrangian of $\operatorname{Kos}\left(x_{1}\right)$ and use the multiplicative structure, as explained in Example 2.12.

Remark 3.4. This result is of course important because it relates the Witt group of two regular schemes $\operatorname{Spec}(R)$ and $U$, with Krull dimension going down by one. This could be of some help, one day, in a proof of Knebusch's conjecture for semilocal regular rings by induction on the Krull dimension. Of course, the scheme $U$ is not semi-local anymore, and its Witt group does not inject in the Witt group 
of its function field in general. To see this, it suffices to construct a (semi-)local ring of dimension congruent to 1 modulo 4 , in which the exact sequence of Theorem 3.3 splits. For instance $R=\kappa\left[X_{1}, \ldots, X_{4 k+1}\right]_{<X_{1}, \ldots, X_{4 k+1}>}$ or $R=$ $\kappa\left[\left[X_{1}, \ldots, X_{4 k+1}\right]\right]$ where $\kappa$ is a field of characteristic different from 2 . It the latter case, one can check that $\mathrm{W}(U)=\mathrm{W}(\kappa)[\epsilon] / \epsilon^{2}$ and that the class $\epsilon$ goes to zero in the Witt group of the field of fractions.

Remark 3.5. Theorem 3.3 was proved for $R$ local regular of dimension $\leq 3$ by Ojanguren, Parimala, Sridharan and Suresh [12], and for higher dimensional such rings in [4]. The above short proof is very much in the spirit of [4]. The proof that $\mathrm{W}^{n}\left(\mathrm{D}^{(d)}(R)\right) \rightarrow \mathrm{W}^{n}(R)$ is the zero map is clarified by the use of the multiplicative structure.

Corollary 3.6. Let $R$ be a noetherian semi-local regular domain which contains $\frac{1}{2}$. Assume that $R$ is of Krull dimension less than or equal to 4 . Let $Q$ be its field of fractions. Then the natural map $\mathrm{W}(R) \rightarrow \mathrm{W}(Q)$ is injective.

Proof. Let $U$ be the punctured spectrum as in Theorem 3.3. Consider the obvious factorization of $\mathrm{W}(R) \rightarrow \mathrm{W}(U) \rightarrow \mathrm{W}(Q)$. The scheme $U$ is regular of dimension 3 , so we know that $\mathrm{W}(U) \rightarrow \mathrm{W}(Q)$ is injective. This was established by Charles Walter and the author in [4, Theorem 10.3]. (This is also an easy consequence of Corollary 2.10.) The statement now follows from Theorem 3.3.

\section{Nilpotence of locally trivial symmetric spaces}

Recall the abbreviated terminology introduced in 2.5 .

Main Lemma 4.1. Let $X$ be a regular scheme containing $\frac{1}{2}$. Let $k \geq 0$ be an integer. Let $\alpha$ and $\beta \in \mathrm{W}(X)$. Assume that $\alpha$ belongs to the image of $\mathrm{W}\left(\mathrm{D}^{(4 k)}(X)\right) \rightarrow \mathrm{W}(X)$ and that $\beta \in \mathrm{W}(X)$ goes to zero in $\mathrm{W}\left(\mathcal{O}_{X, x}\right)$ for all $x \in X^{(4 k)}$. Then $\alpha \cdot \beta \in \mathrm{W}(X)$ belongs to the image of $\mathrm{W}\left(\mathrm{D}^{(4 k+4)}(X)\right) \rightarrow \mathrm{W}(X)$.

Proof. We abbreviate $\mathrm{D}^{(k)}$ for $\mathrm{D}^{(k)}(X)$, since there is only one scheme under consideration. Let $\alpha^{\prime} \in \mathrm{W}\left(\mathrm{D}^{(4 k)}\right)$ such that $\alpha^{\prime} \mapsto \alpha \in \mathrm{W}(X)$. Define $\gamma=\alpha^{\prime} \cdot \beta \in$ $\mathrm{W}\left(\mathrm{D}^{(4 k)}\right)$, using the multiplicative structure (see 2.11). We claim that $\gamma \mapsto 0$ in $\mathrm{W}\left(\mathrm{D}^{(4 k)} / \mathrm{D}^{(4 k+1)}\right)$. Since the map

$$
\mathrm{W}\left(\mathrm{D}^{(4 k)}\right) \rightarrow \mathrm{W}\left(\mathrm{D}^{(4 k)} / \mathrm{D}^{(4 k+1)}\right) \simeq \bigoplus_{x \in X^{(4 k)}} \mathrm{W}\left(\mathrm{D}^{\mathrm{b}}\left(\mathcal{O}_{X, x} \text { - fin.leng. }\right)\right)
$$

is $\mathrm{W}(X)$-linear by 2.11 , it suffices to see that $\beta \in \mathrm{W}(X)$ acts by zero on the righthand side. This is clear since the action of $\mathrm{W}(X)$ on each $\mathrm{W}\left(\mathrm{D}^{\mathrm{b}}\left(\mathcal{O}_{X, x}\right.\right.$ - fin.leng. $\left.)\right)$ 
factors through the ring $\mathrm{W}\left(\mathcal{O}_{X, x}\right)$ where $\beta$ is zero by assumption. This proves $\gamma \mapsto 0$ in $\mathrm{W}\left(\mathrm{D}^{(4 k)} / \mathrm{D}^{(4 k+1)}\right)$.

By the exact sequence $\mathrm{W}\left(\mathrm{D}^{(4 k+1)}\right) \rightarrow \mathrm{W}\left(\mathrm{D}^{(4 k)}\right) \rightarrow \mathrm{W}\left(\mathrm{D}^{(4 k)} / \mathrm{D}^{(4 k+1)}\right)$, we can lift $\gamma$ to some $\gamma^{\prime} \in \mathrm{W}\left(\mathrm{D}^{(4 k+1)}\right)$. By Corollary 2.10 , we can lift $\gamma$ further to $\mathrm{W}\left(\mathrm{D}^{(4 k+4)}\right)$. This proves the Lemma since $\gamma \mapsto \alpha \cdot \beta$ in $\mathrm{W}(X)$.

Theorem 4.2. Let $X$ be a regular scheme containing $\frac{1}{2}$. Assume that $X$ has finite Krull dimension. Then the ideal of locally trivial Witt classes over $X$ is nilpotent. More precisely:

$$
\left(\bigcap_{x \in X} \operatorname{Ker} \mathrm{W}(X) \rightarrow \mathrm{W}\left(\mathcal{O}_{X, x}\right)\right)^{\left[\frac{\operatorname{dim}(X)}{4}\right]+1}=0 .
$$

Proof. This follows easily from the Main Lemma. As before, we abbreviate $\mathrm{D}^{(k)}$ for $\mathrm{D}^{(k)}(X)$. Choose $\alpha \in \bigcap_{x \in X} \operatorname{Ker} \mathrm{W}(X) \rightarrow \mathrm{W}\left(\mathcal{O}_{X, x}\right)$.

From the exact sequence $\mathrm{W}\left(\mathrm{D}^{(1)}\right) \rightarrow \mathrm{W}\left(\mathrm{D}^{(0)}\right) \rightarrow \mathrm{W}\left(\mathrm{D}^{(0)} / \mathrm{D}^{(1)}\right)$ and the identifications $\mathrm{D}^{(0)}=\mathrm{D}^{\mathrm{b}}(X)$ and $\mathrm{D}^{(0)} / \mathrm{D}^{(1)} \simeq \coprod_{x \in X^{(0)}} \mathrm{D}^{\mathrm{b}}\left(\mathcal{O}_{X, x}\right)$, we can lift $\alpha$ to $\mathrm{W}\left(\mathrm{D}^{(1)}\right)$. Now using $\mathrm{W}\left(\mathrm{D}^{(4)}\right) \rightarrow \mathrm{W}\left(\mathrm{D}^{(3)}\right) \simeq \mathrm{W}\left(\mathrm{D}^{(2)}\right) \simeq \mathrm{W}\left(\mathrm{D}^{(1)}\right)$ of Corollary 2.10, we lift our $\alpha$ to $\mathrm{W}\left(\mathrm{D}^{(4)}\right)$. Note that if $\operatorname{dim}(X) \leq 3$, this forces $\alpha=0$. For $X$ of higher Krull dimension, we want to lift $\alpha$ further up to $\mathrm{W}\left(\mathrm{D}^{(4 k)}\right)$, for $k=2,3, \ldots$ By the Main Lemma, this can be done if we multiply $\alpha$ by some Witt class which goes to zero in all $\mathrm{W}\left(\mathcal{O}_{X, x}\right)$ for $x \in X^{(4 k)}$, like for instance any other class in our kernel. Hence we obtain the result by induction on the Main Lemma.

Corollary 4.3. Let $X$ be a regular integral scheme defined over a field of characteristic not 2. Let $Q$ be the function field of $X$. Assume that $X$ has finite Krull dimension. Then

$$
(\operatorname{Ker} \mathrm{W}(X) \rightarrow \mathrm{W}(Q))^{\left[\frac{\operatorname{dim}(X)}{4}\right]+1}=0
$$

Proof. When $X$ is defined over a field, the above kernel coincides with the kernel of Theorem 4.2 since $\mathrm{W}\left(\mathcal{O}_{X, x}\right) \hookrightarrow \mathrm{W}(Q)$ for all $x \in X$, by Ojanguren-Panin [11, Theorem A].

Remark 4.4. Once Knebusch's conjecture will be proved, Corollary 4.3 will be true without assuming the existence of a ground field. For the moment, we record the following secular result. 
Theorem 4.5. Let $X$ be a regular integral scheme containing $\frac{1}{2}$. Let $Q$ be its function field. Assume that $X$ has finite Krull dimension. Then there exists an integer $N \geq 1$ such that $(\operatorname{Ker} \mathrm{W}(X) \rightarrow \mathrm{W}(Q))^{N}=0$. Moreover, we can take $N \leq 2^{\left[\frac{\operatorname{dim}(X)}{4}\right]}$.

Proof. As before, we write $\mathrm{D}^{(k)}$ for $\mathrm{D}^{(k)}(X)$. The result is a simple induction on the following

Claim. For any $k \geq 1$ we have $\left(\operatorname{Im}\left(\mathrm{W}\left(\mathrm{D}^{(4 k)}\right) \rightarrow \mathrm{W}(X)\right)\right)^{2} \subset \operatorname{Im}\left(\mathrm{W}\left(\mathrm{D}^{(4 k+4)}\right) \rightarrow\right.$ $\mathrm{W}(X))$.

This Claim follows from the Main Lemma 4.1 once we know that for any $x \in X^{(4 k)}, k \geq 1$, the natural map $\mathrm{W}\left(\mathrm{D}^{(4 k)}\right) \rightarrow \mathrm{W}\left(\mathcal{O}_{X, x}\right)$ is zero. To see this, let $U_{x}$ be the punctured spectrum of $\mathcal{O}_{X, x}$ and observe that the following composition is zero

$$
\mathrm{W}\left(\mathrm{D}^{(4 k)}(X)\right) \rightarrow \mathrm{W}\left(\mathcal{O}_{X, x}\right) \rightarrow \mathrm{W}\left(U_{x}\right)
$$

because the composition of the underlying morphisms $\mathrm{D}^{(4 k)}(X) \rightarrow \mathrm{D}^{\mathrm{b}}\left(\mathcal{O}_{X, x}\right) \rightarrow$ $\mathrm{D}^{\mathrm{b}}\left(U_{x}\right)$ is already zero on the level of triangulated categories. Now the map $\mathrm{W}\left(\mathcal{O}_{X, x}\right) \rightarrow \mathrm{W}\left(U_{x}\right)$ is injective by Theorem 3.6 or [4, Theorem 9.1]. This finishes the proof of the Claim.

Remark 4.6. It is shown in [6] that the regularity assumption is necessary for these nilpotence results.

\section{The best general exponent}

We want to prove that $N=\left[\frac{\operatorname{dim} X}{4}\right]+1$ is the best exponent in general. To see this, we shall compute a collection of examples. We keep the abbreviated terminology introduced in 2.5 .

Proposition 5.1. (Walter). Let $X$ be a regular scheme which contains $\frac{1}{2}$. Let $n \in \mathbb{Z}$. We have an isomorphism:

$$
\mathrm{W}^{n}\left(\mathbb{P}_{X}^{1}\right) \simeq \mathrm{W}^{n}(X) \oplus \mathrm{W}^{n-1}(X)
$$

such that

(1) This isomorphism is $\mathrm{W}(X)$-linear, with the natural action of $\mathrm{W}(X)$ on both sides (2.11). 
(2) For $n=0$, the usual multiplication on $\mathrm{W}\left(\mathbb{P}_{X}^{1}\right) \simeq \mathrm{W}(X) \oplus \mathrm{W}^{-1}(X)$ becomes on the direct sum: $(a, e) \cdot(b, f)=(a \cdot b, a \cdot f+b \cdot e)$; in particular, $(0, e) \cdot(0, f)=$ 0 .

(3) Again for $n=0$, if we assume moreover that $X$ is integral, the summand $\mathrm{W}^{-1}(X)$ goes to zero via the homomorphism $\mathrm{W}\left(\mathbb{P}_{X}^{1}\right) \rightarrow \mathrm{W}(Q)$ where $Q$ is the function field of $\mathbb{P}_{X}^{1}$.

Proof. This isomorphism will appear in Walter [14] and is already in Gille [7, Theorem 5.4] for $X$ affine. We sketch the gross strategy of the proof here for the convenience of the reader. Consider the closed subscheme $Z_{0}$ of $\mathbb{P}_{X}^{1}$ given by the image of the zero section $i_{0}: X \rightarrow \mathbb{P}_{X}^{1}$. Its open complement $U$ is an affine line over $X, U \simeq \mathbb{A}_{X}^{1}$, and by [3, Theorem 3.4], $\mathrm{W}^{n}(U) \simeq \mathrm{W}^{n}(X)$, for instance via the natural map $U \rightarrow \mathbb{P}_{X}^{1} \rightarrow X$. Therefore the homomorphism $\mathrm{W}^{n}\left(\mathbb{P}_{X}^{1}\right) \rightarrow \mathrm{W}^{n}(U)$ is split surjective. The result follows essentially from the Localization Theorem 2.3 applied to $\mathbb{P}_{X}^{1}$ and the open $U$. The main difficulty is to establish the non-trivial isomorphism $\mathrm{W}^{n-1}(X) \stackrel{\sim}{\rightarrow} \mathrm{W}_{Z_{0}}^{n}\left(\mathbb{P}_{X}^{1}\right)$; this is done in loc. cit. It is obvious that $\mathrm{W}_{Z_{0}}^{n}\left(\mathbb{P}_{X}^{1}\right)$ goes to zero in the function field of $\mathbb{P}_{X}^{1}$ because it is made of elements supported on a proper closed subscheme of $\mathbb{P}_{X}^{1}$. To see $(2)$, it essentially amounts to prove that the product of two elements in $\operatorname{Ker}\left(\mathrm{W}\left(\mathbb{P}_{X}^{1}\right) \rightarrow \mathrm{W}(U)\right)$ is zero in $\mathrm{W}\left(\mathbb{P}_{X}^{1}\right)$. This is true since we could use another section $X \rightarrow \mathbb{P}_{X}^{1}$ in the above localization argument, for instance $i_{1}: X \rightarrow \mathbb{P}_{X}^{1}$. The image in $\mathrm{W}\left(\mathbb{P}_{X}^{1}\right)$ of both Witt groups with supports $\mathrm{W}_{Z_{0}}\left(\mathbb{P}_{X}^{1}\right)$ and $\mathrm{W}_{Z_{1}}\left(\mathbb{P}_{X}^{1}\right)$ are isomorphic by homotopy invariance, since both the 0 -section and the 1 -section can be included in an affine line $\mathbb{A}_{X}^{1}$, for instance outside $\infty$. Clearly, the product in $W\left(\mathbb{P}_{X}^{1}\right)$ of an element coming from $\mathrm{W}_{Z_{0}}\left(\mathbb{P}_{X}^{1}\right)$ with an element coming from $\mathrm{W}_{Z_{1}}\left(\mathbb{P}_{X}^{1}\right)$ is zero since it will come from $\mathrm{W}_{Z_{0} \cap Z_{1}}\left(\mathbb{P}_{X}^{1}\right)=\mathrm{W}_{\emptyset}\left(\mathbb{P}_{X}^{1}\right)=0$. Details will be found in [14], and moreover for arbitrary projective bundles.

Lemma 5.2. Let $X$ be a regular integral scheme containing $\frac{1}{2}$. Assume that there is an integer $m \geq 2$ and an element $\alpha \in \mathrm{W}(X)$ which satisfy the following conditions:

(1) The element $\alpha$ goes to zero in the Witt group of the function field of $X$.

(2) The element $\alpha^{m-1}$ is not torsion in $\mathrm{W}(X)$, that is for $n \in \mathbb{Z}, n \cdot \alpha^{m-1}=$ $0 \Rightarrow n=0$.

(3) We have $\alpha^{m}=0$.

Let $Y:=\mathbb{P}^{1} \times \mathbb{P}^{1} \times \mathbb{P}^{1} \times \mathbb{P}^{1} \times X$. Then there is an element $\beta \in \mathrm{W}(Y)$ which goes to zero in the Witt group of the function field of $Y$, such that $\beta^{m+1}=0$ and such that $\beta^{m}$ is not torsion; in particular $\beta^{m} \neq 0$.

Proof. Apply four times the above Proposition. We have

$$
\mathrm{W}\left(\mathbb{P}^{1} \times \mathbb{P}^{1} \times \mathbb{P}^{1} \times \mathbb{P}^{1} \times X\right) \simeq \mathrm{W}(X) \oplus \text { Blabla } \oplus \mathrm{W}^{-4}(X)
$$

where Blabla is a sum of copies of $\mathrm{W}^{-1}(X), \mathrm{W}^{-2}(X), \mathrm{W}^{-3}(X)$, respectively 4,6 
and 4 times. Observe that $\mathrm{W}^{-4}(X) \cong \mathrm{W}(X)$ and thus we can define $\beta:=(\alpha, 0,1)$ where $0 \in$ Blabla and $1 \in \mathrm{W}^{-4}(X) \cong \mathrm{W}(X)$ is the unit element. Using (1) and (2) of the above Proposition, we see immediately by induction on $j \in \mathbb{N}$ that $\beta^{j}=\left(\alpha^{j}, 0, j \cdot \alpha^{j-1}\right)$. Therefore $\beta^{m+1}=0$ whereas $\beta^{m}=\left(0,0, m \cdot \alpha^{m-1}\right)$. The result follows. Note that $\beta$ goes to zero in the function field of $Y$ by (3) of the above Proposition.

Corollary 5.3. The exponent $\left[\frac{\operatorname{dim}(X)}{4}\right]+1$ is the best general exponent which satisfies the nilpotence assertion of Corollary 4.3.

Proof. Clearly, $\operatorname{dim}\left(\mathbb{P}^{1} \times \mathbb{P}^{1} \times \mathbb{P}^{1} \times \mathbb{P}^{1} \times X\right)=\operatorname{dim}(X)+4$. The result follows by induction on the Krull dimension, using the above Lemma. It suffices to find a starting point, i.e. a regular integral scheme $X$ of Krull dimension 4 , and an element $\alpha \in \mathrm{W}(X)$ which goes to zero in the Witt group of the function field but which is not torsion (i.e. $m=2$ in the hypotheses of the Lemma). Take for instance $X=\mathbb{P}^{1} \times \mathbb{P}^{1} \times \mathbb{P}^{1} \times \mathbb{P}^{1} \times \operatorname{Spec}(\mathbb{R})$. The above proof shows that $\mathrm{W}(X)=\mathbb{Z}[\epsilon] / \epsilon^{2}$ and that $\epsilon \mapsto 0$ in the Witt group of the function field of $X$. We choose of course $\alpha=\epsilon$.

Remark 5.4. We could have cooked up a more explicit example, without referring to the yet unpublished [14], replacing some general facts by ad hoc arguments. Nevertheless, the proof of Theorem 4.2, in which a new obstruction obviously pops up in every fourth dimension, is probably enough to convince the reader that $N=\left[\frac{\operatorname{dim}(X)}{4}\right]+1$ is the best general exponent which kills the kernel. Therefore, we prefer to give here some large class of examples, in the reader's obvious scientific interest.

Remark 5.5. In this paper, we have constructed two classes of examples of regular integral schemes $X$ of dimension 4 (and higher) with non-trivial kernel. The first is the punctured spectrum of any 5-dimensional regular semi-local ring (see 3.4). The second is $\mathbb{P}_{\kappa}^{1} \times \mathbb{P}_{\kappa}^{1} \times \mathbb{P}_{\kappa}^{1} \times \mathbb{P}_{\kappa}^{1}$ for any field $\kappa$ of characteristic different from 2.

\section{Nilpotence of shifted Witt groups}

We keep the abbreviated terminology of 2.5 .

Proposition 6.1. Let $X$ be a regular scheme containing $\frac{1}{2}$. Assume that $X$ is of finite Krull dimension. Consider the summand $\mathrm{W}^{\prime}:=\mathrm{W}^{1}(X) \oplus \mathrm{W}^{2}(X) \oplus \mathrm{W}^{3}(X)$ in the total Witt group $\mathrm{W}^{\text {tot }}:=\mathrm{W}(X) \oplus \mathrm{W}^{1}(X) \oplus \mathrm{W}^{2}(X) \oplus \mathrm{W}^{3}(X)$, which is a $\mathbb{Z} / 4$-graded ring with either the left or the right multiplication (see 2.11). Then the $N^{\prime}$-th power of $\mathrm{W}^{\prime}$ in $\mathrm{W}^{\text {tot }}$ is zero, where $N^{\prime}=4 \cdot\left(\left[\frac{\operatorname{dim} X}{4}\right]+1\right) \leq \operatorname{dim}(X)+4$. 
Proof. Denote by $J(X)=\bigcap_{x \in X} \operatorname{Ker}\left(\mathrm{W}^{0}(X) \rightarrow \mathrm{W}^{0}\left(\mathcal{O}_{X, x}\right)\right)$ the ideal of locally trivial Witt classes in $\mathrm{W}^{0}(X)$. Denote by $J^{\prime}(X)$ the ideal of $\mathrm{W}^{\text {tot }}$ generated by $J(X)$. By Theorem 4.2, we have $J^{\prime}(X)^{N}=0$, where $N=\left[\frac{\operatorname{dim} X}{4}\right]+1$, so it clearly suffices to prove that $\left(\mathrm{W}^{\prime}\right)^{4} \subset J^{\prime}(X)$.

Let $i_{1}, i_{2}, i_{3}, i_{4} \in\{1,2,3\}$ and let $\alpha_{j} \in \mathrm{W}^{i_{j}}(X)$ for $j=1, \ldots, 4$. There is necessarily a non-empty subset $E \subset\{1, \ldots, 4\}$ such that $\sum_{j \in E} i_{j} \equiv 0 \bmod 4$. This can be checked by inspection. To get our result it suffices to see that $\prod_{j \in E} \alpha_{j} \in$ $J(X)$.

This is immediate. The product belongs to $\mathrm{W}^{0}(X)$ by choice of $E$. Using the naturality of the product structure (see details in [8, Section 3.2]), it suffices to see that each $\alpha_{j}$ goes to zero in $\mathrm{W}^{i_{j}}\left(\mathcal{O}_{X, x}\right)$ for any $x \in X$. This holds because shifted Witt groups of local rings vanish: $\mathrm{W}^{n}\left(\mathcal{O}_{X, x}\right)=0$ when $n=1,2,3$, see [2, Theorem 5.6].

\section{References}

[1] P. Balmer, Triangular Witt groups, Part I: The 12-term localization exact sequence, $K$ theory 19, no. 4 (2000), 311-363.

[2] P. Balmer, Triangular Witt groups, Part II: From usual to derived, Math. Z. 236, no. 2 (2001), 351-382.

[3] P. Balmer, Witt cohomology, Mayer-Vietoris, homotopy invariance and the Gersten Conjecture, K-theory 23, no. 1 (2001), 15-30.

[4] P. Balmer and Ch. Walter, A Gersten-Witt spectral sequence for regular schemes, Ann. Sci. École Norm. Sup. (4) 35, no. 1 (2002), 127-152.

[5] A. Beilinson, J. Bernstein et P. Deligne, Faisceaux Pervers, Astérisque 100, Société Mathématique de France (1982).

[6] T. C. Craven, A. Rosenberg and R. Ware, The map of the Witt ring of a domain into the Witt ring of its field of fractions, Proc. Amer. Math. Soc. 51 (1975), 25-30.

[7] S. Gille, On Witt groups with support, Math. Ann. 322, no. 1 (2002), 103-137.

[8] S. Gille and A. Nenashev, Pairings in triangular Witt theory, J. Algebra (to appear).

[9] M. Knebusch, Symmetric bilinear forms over algebraic varieties, in: Conf. on Quadratic Forms, Kingston 1976, 103-283, Queen's Papers on Pure and Applied Mathematics 46, 1977.

[10] M.-A. Knus, Quadratic and hermitian forms over rings, Grundlehren der Mathematischen Wissenschaften 294, Springer Verlag, Berlin, 1991.

[11] M. Ojanguren and I. Panin, A purity theorem for the Witt group, Ann. Sci. École Norm. Sup. (4), 32, no. 1 (1999), 71-86.

[12] M. Ojanguren, R. Parimala, R. Sridharan and V. Suresh, Witt groups of the punctured spectrum of a 3-dimensional regular local ring and a purity theorem, J. London Math. Soc. (2) 59, no. 2 (1999), 521-540.

[13] J.-L. Verdier, Catégories dérivées des catégories abéliennes, Astérisque 239, Société mathématique de France (1996).

[14] Ch. Walter, Witt groups of projective bundles, Talk at the March 2001 Münster Workshop on Witt groups of algebraic varieties, article in preparation.

[15] Ch. Weibel, An introduction to homological algebra, Cambridge University Press, 1994. 
Paul Balmer

Department of Mathematics

ETHZ

8092 Zürich

Switzerland

e-mail: balmer@math.ethz.ch

(Received: December 4, 2001)

(1) To access this journal online:

(4) http://www.birkhauser.ch 